\title{
Frequency-Hopping Signal Network-Station Sorting Based on Maxout Network Model and Generative Method
}

\author{
Hongguang Li $\mathbb{D},{ }^{1}$ Ying Guo, ${ }^{1}$ Ping Sui $\mathbb{D},{ }^{1}$ Xinyong Yu, ${ }^{2}$ Xin Yang, ${ }^{1}$ and Shaobo Wang ${ }^{1}$ \\ ${ }^{1}$ Institute of Information and Navigation, Air Force Engineering University, Xian, Shaanxi, 710077, China \\ ${ }^{2}$ Air Force Communication NCO Academy, Dalian, Shenyang, 116600, China
}

Correspondence should be addressed to Hongguang Li; toumingwings@163.com

Received 26 February 2019; Accepted 23 May 2019; Published 4 June 2019

Academic Editor: Fazal M. Mahomed

Copyright (C) 2019 Hongguang Li et al. This is an open access article distributed under the Creative Commons Attribution License, which permits unrestricted use, distribution, and reproduction in any medium, provided the original work is properly cited.

Automatic frequency-hopping (FH) signal network-station sorting is one of the most difficult and import problems in the field of electronic warfare, especially in a complex electromagnetic environment. In this paper, an automatic and reliable network-station sorting method of FH signal with maxout network feature extraction and generative-based classification method is proposed. Experiments on real FH data sets demonstrate that the proposed method not only outperforms the competitive feature extraction methods with a higher accuracy of FH signal network-station sorting but also has a better robustness against noise, especially Gaussian noise.

\section{Introduction}

Frequency-hopping (FH) communication has become an important means of counter reconnaissance and antijamming in military field and been widely applied in military and civilian communications because of its low probability of interception, antijamming performance, networking capability, and inherent security feature. At the same time, these characteristics also pose a serious challenge to FH communication's application on radio reconnaissance.

The network-station sorting of the frequency-hopping (FH) signal refers to separating the hop signals corresponding to all or a specific network station from the mixed signals of the multiple FH network stations, which is a difficult point in the field of FH signal detection and processing. The technology of network-station sorting of FH signals is prosperous in the communication countermeasure and radio-monitoring domain. How to quickly and effectively realize the FH signal network-station sorting becomes a severe challenge in signal processing.

To address this problem, a number of works have appeared in literatures [1-7]. In view of those methods, most of them consider two steps, i.e., feature extraction and classification, which resulted in that the extracted FH signal features may be very crucial in sorting accuracy of FH signal network-station.

Due to the device dispersion of the $\mathrm{FH}$ station and the inconsistency in the manufacturing process, it comes with unavoidable subtle differences of FH signals from each radio transmitter, which are generally generated during the production process of their component, e.g., filters, power amplifiers, and digital-to-analog converters. Especially in the radio frequency part, owing to the use of analog devices, it will inevitably lead to subtle differences in the $\mathrm{FH}$ radio, which reflect the individual feature of the station uniquely. These subtle differences are also called radio frequency (RF) fingerprints of $\mathrm{FH}$ radio stations. Through RF fingerprint, it is possible to discriminate radio emitters.

Based on the different types of RF fingerprint feature extraction, current feature extraction method of FH signal can be classified into two main categories: transient feature extraction and steady-state feature extraction [8-10]. Considering the fact that when a $\mathrm{FH}$ station is activated or "keyed," it goes through a relatively short transient phase during which the $\mathrm{FH}$ signal emanating from the unit displays characteristics that are believed to be unique to the extent that they can be used to unambiguously identify an individual FH station. Individual feature analysis of steady-state signals 


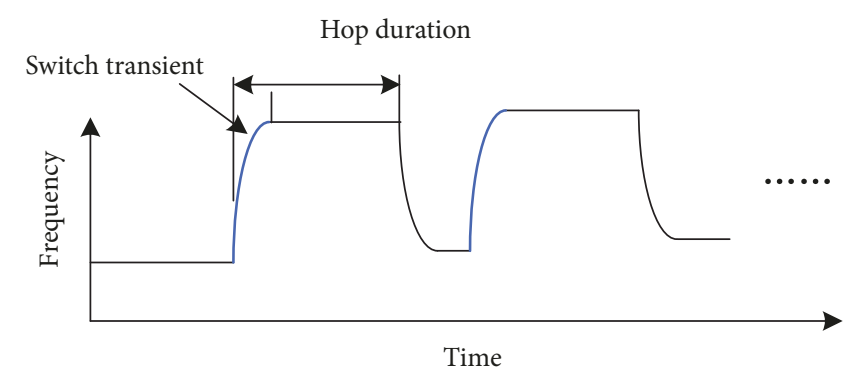

FIGURE 1: Signal transmission schematic diagram of FH station.

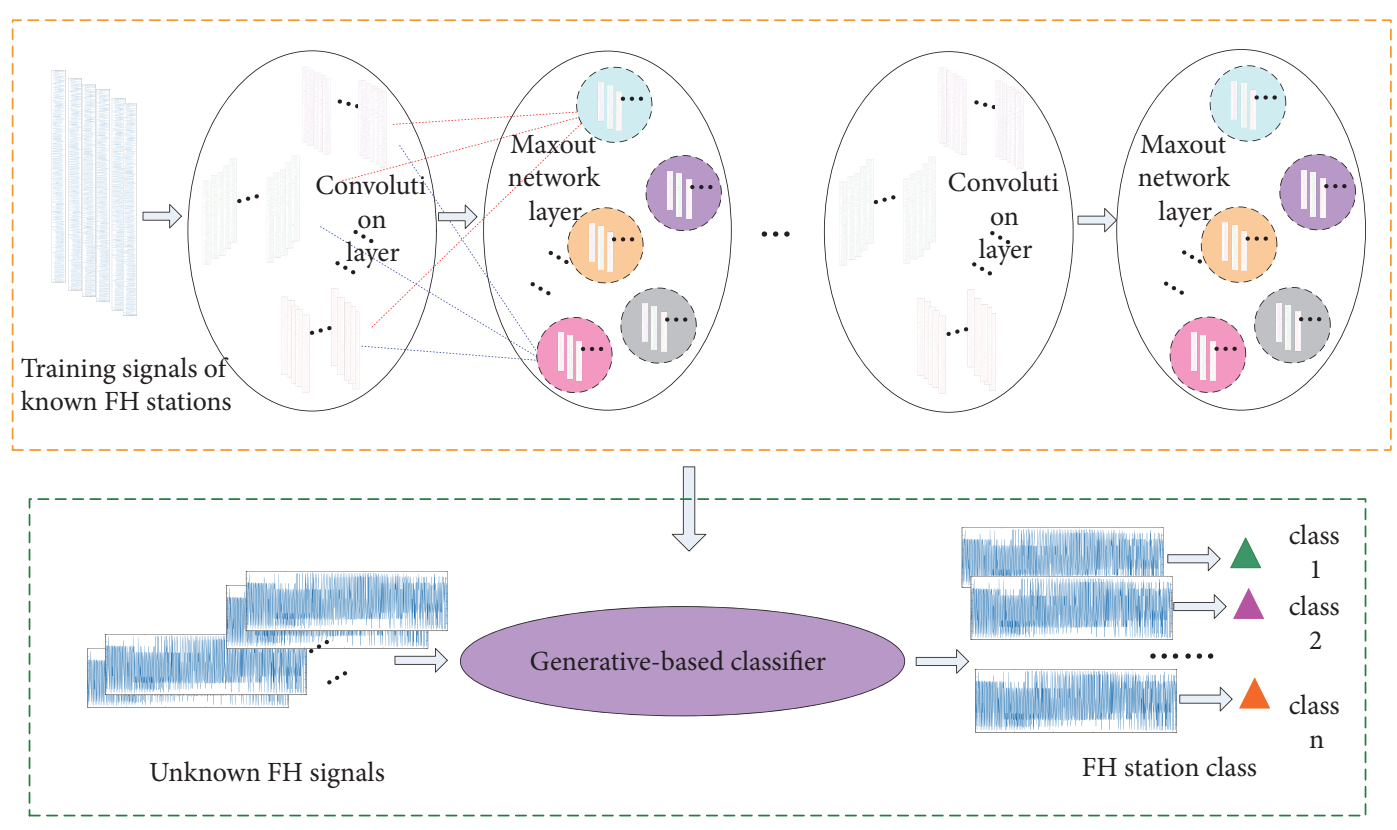

FIgURE 2: The FH signal network-station sorting process of our method.

is more difficult than transient signal feature extraction [10]. Under steady-state operation, the internal device differences of the station are expressed in the form of "synthesis." Therefore, it is difficult to mathematically model the influence of internal variations of all devices on the signal. Interesting, the main characteristic of FH signal is the pseudo-random variance of carrier frequencies according to a certain hopping pattern. It takes a certain amount of time to adjust the signal from one frequency point to another, which makes each hop signal have a switch transient moment before it is established, as shown in Figure 1. Therefore, hopping switch transient analysis can be used to analyze the FH signal. Motivated by this fact, this paper proposes a new FH signal network-station sorting method that is based on maxout network and generative method. FH signals generated by five kinds of stations are collected and analyzed. Maxout network model is used to acquire the fingerprint characteristics of the FH signal, based on the transient feature extraction of the FH switch transient analysis. Generative method optimized by expectation maximization (EM) optimization algorithm is employed as classifier to realize the networkstation sorting of the five kinds of FH station. Results show that the proposed method can effectively improve the sorting accuracy of FH network-station. Although our implementation deals exclusively with FH signal networkstation sorting, the approach itself is general and will work with any identical radio sources recognition scheme. Procedures of FH signal network-station sorting based on maxout network model and generative method are illustrated in Figure 2.

More specially, this paper is organized as follows: in Section 2, we briefly review some related feature learning methods, which motivated our research. In Section 3, we describe our FH signal fingerprint feature extraction method according to the maxout network model and the networkstation sorting algorithm by generative method, whereas in Section 4, we make an evaluation of performance and present some comparison results. Finally, in Section 5 we draw conclusions.

\section{Related Work}

The main goal of feature extraction is to convert FH transient signal into its distinctive feature space, making the $\mathrm{FH}$ 
signal sorting process easily. Recently, deep neural networks have been widely used in pattern classification and feature extraction because of its formidable generalization ability brought by its inherent ability to fit complex functions and its powerful characteristic representation from multiparameters and high dimensionality [11].

By training features on very large datasets, several deeplearning methods achieved very high pattern recognition rates. The convolutional neural network $(\mathrm{CNN})$ is one of the state-of-the-art learning methods for pattern recognition. DCNN as a deeper model of CNN has been designed to automatically transform input data into its distinct feature space. Although this method can be more successful than traditional pattern recognition method in recognition rate due to its deep architecture, running this model is extremely time consuming because of its multiparameters and high dimensionality. In 2013, Goodfelow [12] proposed a maxout network model, which can reduce the computational costs by minimizing the number of necessary neurons and the number of network parameters in each maxout layer. In 2015, Schroff [13] successfully applied maxout networks in face recognition, which gain high recognition accuracy rates on challenging face datasets. Inspired by these deep-learning techniques, the maxout network is designed as a real-time distinctive features extraction model. In addition, the generative method is combined to realize the FH signal networkstation sorting finally. The detailed proposed approach is as follows.

\section{Proposed Approach}

3.1. Fingerprint Feature Learning with Maxout Network. The maxout model is simply a feed-forward architecture, such as a multilayer perceptron or deep convolutional neural network, which uses a new type of activation function: the maxout unit [12]. Given an input data $x \in \mathbb{R}^{d}$, where $x$ may be an original data, or may be a hidden layer's state, the function of a maxout hidden layer is given by

$$
g_{v}(x)=\max _{r \in[1, k]} z_{v r}
$$

where $z_{v r}=x^{T} W_{v r}+b_{v r}, W \in \mathbb{R}^{d \times m \times k}$, and $b_{v r} \in \mathbb{R}^{m \times k}$ are learned parameters, in which $d$ is the dimension of input data, $m$ is the number of hidden layer nodes, and $k$ denotes that each hidden layer node expands $k$ intermediate nodes, which are all linear output, and each node of maxout takes the maximum output value of the $k$ intermediate nodes. In a convolutional network, a single maxout unit can be interpreted as making a piecewise linear approximation to an arbitrary convex function. Maxout networks learn not just the relationship between hidden units, but also the activation function of each hidden unit [12]. See Figure 2 for a graphical depiction of how this works. Because of the intrinsic superiority of the maxout network in arbitrary convex function approximation, it can achieve excellent performance in fingerprint feature learning.

3.2. FH Signal Network-Station Sorting with Generative Method. For the extracted fingerprint features, this paper uses the generative method to realize the network-station sorting of FH signal. Given feature set $\mathbf{x}$, assume it belongs to station $h \in H$, where $H=\{1,2, \cdots, C\}$ is all possible station classes. This paper assumes that the feature data are generated by a Gaussian mixture model (GMM), and each networkstation class corresponds to a Gaussian mixture component. In other words, the feature data is generated based on the following probability density.

$$
p(\mathbf{x})=\sum_{i=1}^{C} \alpha_{i} \cdot p\left(\mathbf{x} \mid \mu_{i}, \Sigma_{i}\right)
$$

where mixing coefficient $\alpha_{i} \geq 0, \sum_{i=1}^{C} \alpha_{i}=1, p\left(\mathbf{x} \mid \mu_{i}, \Sigma_{i}\right)$ is the probability that feature set $\mathbf{x}$ belongs to the $i$-th $\mathrm{FH}$ network-station and $\mu_{i}$ and $\Sigma_{i}$ are the parameters of the Gaussian mixture component.

Let $f(\mathbf{x}) \in \mathrm{H}$ denote the predictive network-station class of function $f(\cdot)$ to $\mathbf{x}$ and $\Theta \in\{1,2, \cdots, C\}$ the networkstation class of feature set $\mathbf{x}$, which is known by maximizing a posteriori probability

$$
\begin{aligned}
f(\mathbf{x}) & =\underset{j \in \mathrm{H}}{\operatorname{argmax}} p(h=j \mid \mathbf{x}) \\
& =\underset{j \in \mathrm{H}}{\operatorname{argmax}} \sum_{i=1}^{C} p(h=j, \Theta=i \mid \mathbf{x}) \\
& =\underset{j \in \mathrm{H}}{\operatorname{argmax}} \sum_{i=1}^{C} p(h=j \mid \Theta=i, \mathbf{x}) \cdot p(\Theta=i \mid \mathbf{x})
\end{aligned}
$$

where

$$
p(\Theta=i \mid \mathbf{x})=\frac{\alpha_{i} \cdot p\left(\mathbf{x} \mid \mu_{i}, \Sigma_{i}\right)}{\sum_{i=1}^{C} \alpha_{i} \cdot p\left(\mathbf{x} \mid \mu_{i}, \Sigma_{i}\right)}
$$

in which $p(\Theta=i \mid \mathbf{x})$ is the a posteriori probability that the feature set belongs to the $i$-th FH network-station, and $p(h=j \mid \Theta=i, \mathbf{x})$ denotes the probability that the feature set $\mathbf{x}$ belongs to the $i$-th FH network-station but its predictive network-station class is $j$. As we can see, if and only if $i=$ $j, p(h=j \mid \Theta=i)=1$; otherwise $p(h=j \mid \Theta=i)=0$.

Given the feature data $\left\{\mathbf{F}_{l}, \mathbf{F}_{u}\right\}$, where $\mathbf{F}_{l}=\left\{\left(\mathbf{x}_{1}, h_{1}\right)\right.$, $\left.\left(\mathbf{x}_{2}, h_{2}\right), \cdots,\left(\mathbf{x}_{l}, h_{l}\right)\right\}$ represents the feature set of known network-station classes and $\mathbf{F}_{u}=\left\{\left(\mathbf{x}_{l+1}, h_{l+1}\right),\left(\mathbf{x}_{l+2}, h_{l+2}\right), \cdots\right.$, $\left.\left(\mathbf{x}_{u}, h_{u}\right)\right\}$ represents the unknown feature set, where $l \ll u, l+$ $u=M$, as mentioned above, this paper assumes that all feature data are independent and identically distributed and are generated by the same GMM. The parameters of GMM $\left\{\left(\alpha_{i}, \mu_{i}, \Sigma_{i}\right), 1 \leq \mathrm{i} \leq C\right\}$ are estimated by maximum likelihood method

$$
\begin{aligned}
& L\left(\mathbf{F}_{l} \cup \mathbf{F}_{u}\right)=\sum_{\left(x_{j}, h_{j}\right) \in \mathbf{F}_{l}} \ln \left(\sum_{i=1}^{C} \alpha_{i} \cdot p\left(\mathbf{x}_{j} \mid \mu_{i}, \Sigma_{i}\right)\right. \\
& \left.\cdot p\left(h_{j} \mid \Theta=i, \mathbf{x}_{j}\right)\right)+\sum_{x_{j} \in \mathbf{F}_{u}} \ln \left(\sum_{i=1}^{C} \alpha_{i}\right. \\
& \left.\cdot p\left(\mathbf{x}_{j} \mid \mu_{i}, \Sigma_{i}\right)\right)
\end{aligned}
$$




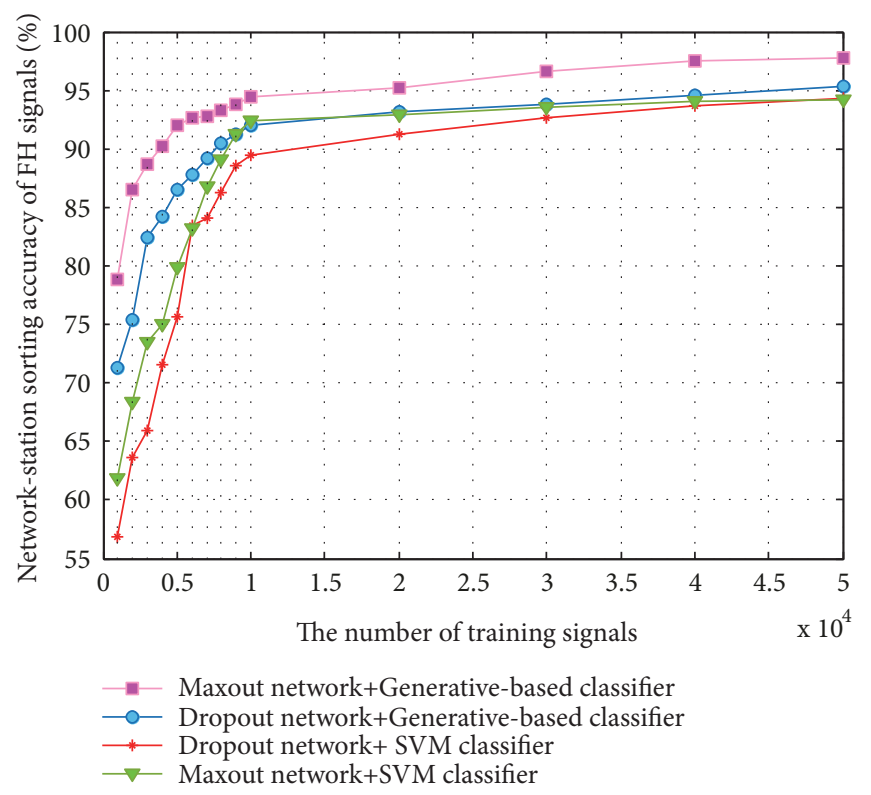

FIGURE 3: FH signal network-station sorting accuracy with different training numbers.

where $L\left(\mathbf{F}_{l} \cup \mathbf{F}_{u}\right)$ denotes $\mathbf{F}_{l} \cup \mathbf{F}_{u}$ 's logarithmic likelihood function, which consists of two items: known FH networkstation class features and unknown network-station class features. Then the above model parameters can be solved by EM algorithm [14], and the iterative update algorithm is as follows

\subsubsection{Expectation Step}

$$
\gamma_{j i}=\frac{\alpha_{i} \cdot p\left(\mathbf{x}_{j} \mid \mu_{i}, \Sigma_{i}\right)}{\sum_{i=1}^{C} \alpha_{i} \cdot p\left(\mathbf{x}_{j} \mid \mu_{i}, \Sigma_{i}\right)}
$$

where $\gamma_{j i}$ denotes the probability that the unknown networkstation feature set $\mathbf{x}_{j}$ belongs to each network-station class.

\subsubsection{Maximization Step}

$$
\begin{aligned}
\mu_{i} & =\frac{1}{\sum_{\mathbf{x}_{j} \in \mathbf{F}_{u}} \gamma_{j i}+l_{i}}\left(\sum_{\mathbf{x}_{j} \in \mathbf{F}_{u}} \gamma_{j i} \mathbf{x}_{j}+\sum_{\left(\mathbf{x}_{j}, h_{j}\right) \in \mathbf{F}_{l} \wedge h_{j}=i} \mathbf{x}_{j}\right) \\
\Sigma_{i} & =\frac{1}{\sum_{\mathbf{x}_{j} \in \mathbf{F}_{u}} \gamma_{j i}+l_{i}}\left(\sum_{\mathbf{x}_{j} \in \mathbf{F}_{u}} \gamma_{j i}\left(\mathbf{x}_{j}-\mu_{i}\right)\left(\mathbf{x}_{j}-\mu_{i}\right)^{T}\right. \\
& \left.+\sum_{\left(\mathbf{x}_{j}, h_{j}\right) \in \mathbf{F}_{i} \wedge h_{j}=i}\left(\mathbf{x}_{j}-\mu_{i}\right)\left(\mathbf{x}_{j}-\mu_{i}\right)^{T}\right) \\
\alpha_{i} & =\frac{1}{m}\left(\sum_{\mathbf{x}_{j} \in \mathbf{F}_{u}} \gamma_{j i}+l_{i}\right)
\end{aligned}
$$

where $l_{i}$ denotes the number of feature sets for known network-stations in class $i$. After the model parameters are obtained by iterative convergence of the above algorithm, the classification and recognition of unknown network-station feature sets can be realized by using formula (3) and (4).

By replacing the Gaussian mixture model in the above process with the mixture expert model [15], the naive Bayesian model [14] can be used to derive other generative learning methods.

\section{Evaluation of Performance}

After the successful FH signal fingerprint feature extraction and generative classification process, the scheme must discriminate the FH transmitters of the FH signals. To test the feature extraction and network-station sorting together, a set of 60000 real FH signals was used. They were distributed approximately among 5 different $\mathrm{FH}$ transmitters, each transmitter has $12000 \mathrm{FH}$ signal.

4.1. Sorting Result with Different Number of Training Signals. In this section, we investigate the influence of the number of training signals on the performance of this paper's proposed method. To train the maxout network, the number of 1000 , $2000, \cdots, 10000$ labeled signals arbitrarily taken from each transmitter was used to train the network; the remaining signals are used for testing. The average result of one hundred individual experiments with different training numbers is shown in Figure 3. From the result we can learn that this paper's method has higher sorting accuracy than other methods. The reason is the maxout network model's powerful characteristic representation from multiparameters and high dimensionality. And with the increase of the training numbers, the sorting rate increases, and when the number of training signals is more than 10000 , the sorting rate changes slowly. 


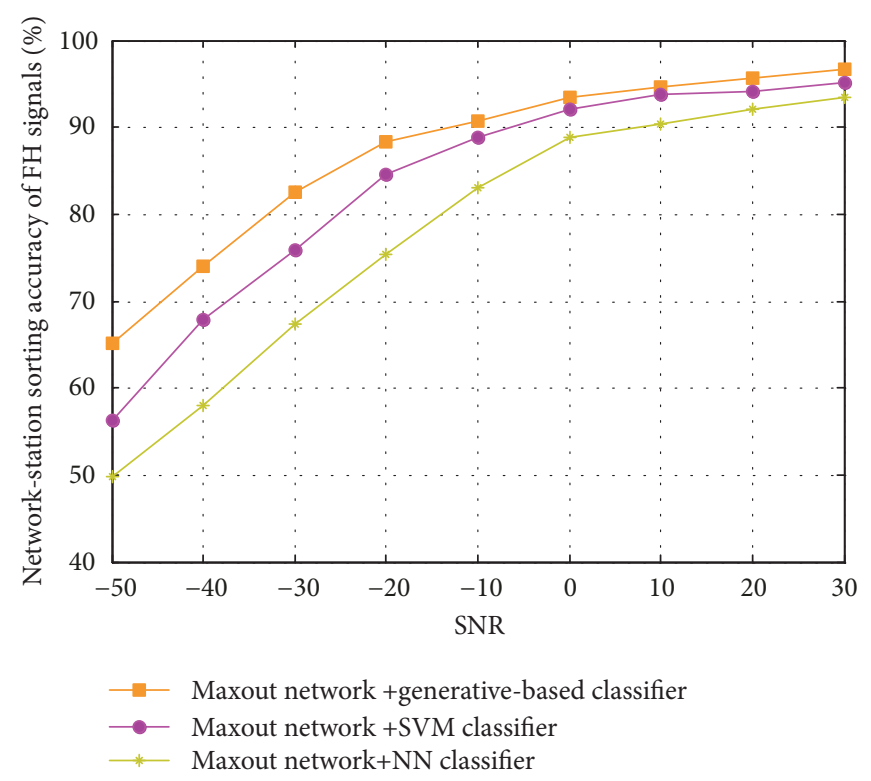

FIGURE 4: FH signal network-station sorting accuracy with different SNRs.

4.2. Robustness of Our Method to Noises. Considering the fact that the FH signals obtained in the actual electronic environment are greatly affected by the environmental noise, however, the acquisition environment of the $\mathrm{FH}$ signals in this paper is relatively clean, and there is no obvious noise. To test the impact of noise on robustness in sorting result for $\mathrm{FH}$ signals, in this test we mixed the obtained FH signals with Gaussian white noise of different intensity to simulate the onsite circumstance. As mentioned above, we have 12000 signals of each FH station, 50\% signals are randomly chosen $t$ as training samples; the rest $50 \%$ signals added with different intensity Gaussian white noise are selected as testing samples. We use the signal-to-noise ratio (SNR) to reflect the intensity of noise, which is presented as follows:

$$
S N R=10 \log _{10}\left(\frac{\sum_{n=1}^{N} S g^{2}(n)}{\sum_{n=1}^{N} N i^{2}(n)}\right)
$$

where $S g(n)$ is the original FH signal, $N i(n)$ is interference noise, and $N$ is the length of the two signals. The length of original signal in this paper is 1024, and the results are shown in Figure 4. From Figure 4 we can see that the results of our method are stable for Gaussian white noise of different intensity because of the robustness of the Gaussian mixture model to noise.

4.3. Robustness of Noise with Different Training Numbers. Then we test the noise robustness with different training number of FH signals. As previously mentioned, we have 12000 signals of each FH station, 1000, 2000, .., 10000 of which are selected as training samples, and the remaining signals added with Gaussian white noise are selected as testing samples at random. The experimental result is shown in Figure 5. From Figures 5 and 4 we can see that the results of our method are robustness to noise in a certain range of SNR.

\section{Conclusion}

In this paper, we propose a novel FH signal network-station sorting method. Firstly, our method takes advantage of the deep neural network, which is maxout network model in this paper, to learn the fingerprint feature of each $\mathrm{FH}$ station; thus the network-station sorting accuracy is improved by the powerful feature representation capability of the maxout network model. And then the maxout network's outputs regard as the FH station's features are used as inputs to the generative-based classifier to realize the $\mathrm{FH}$ signals' networkstation sorting. Experimental results on 5 real work FH stations show that our method achieves obviously better performance than other state-of-the-art methods in terms of accuracy.

\section{Data Availability}

The experimental data of my manuscript is the common result of my laboratory. It is still in the research stage and individuals do not have public rights.

\section{Conflicts of Interest}

The authors declare that there is no conflict of interest regarding the publication of this paper.

\section{Acknowledgments}

The authors are grateful to the support from the National Natural Science Foundation of China (Grant no. 61601500, no. 61871396). 


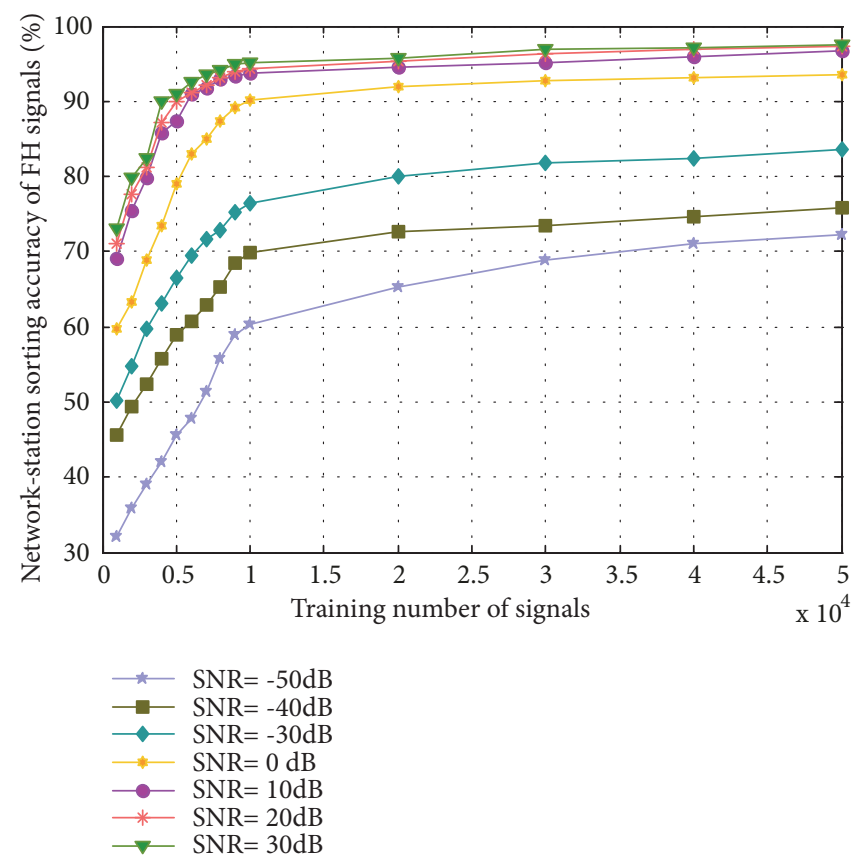

FIGURE 5: FH signal network-station sorting accuracy with different SNRs and training numbers.

\section{References}

[1] Y. Yuan, Z. Huang, H. Wu, and X. Wang, "Specific emitter identification based on Hilbert-Huang transform-based timefrequency-energy distribution features," IET Communications, vol. 8, no. 13, pp. 2404-2412, 2014.

[2] P. Scanlon, I. O. Kennedy, and Y. Liu, "Feature extraction approaches to RF fingerprinting for device identification in femtocells," Bell Labs Technical Journal, vol. 15, no. 3, pp. 141152, 2010.

[3] P. Padilla, J. L. Padilla, and J. F. Valenzuela, "Radiofrequency identification of wireless devices based on RF fingerprinting," IEEE Electronics Letters, vol. 49, no. 22, pp. 1409-1410, 2013.

[4] T. J. Bihl, K. W. Bauer, and M. A. Temple, "Feature selection for RF fingerprinting with multiple discriminant analysis and using ZigBee device emissions," IEEE Transactions on Information Forensics and Security, vol. 11, no. 8, pp. 1862-1874, 2016.

[5] Y. Jia, J. H. Ma, and L. Gan, "Radiometric identification based on low-rank representation and minimum prediction error regularization," IEEE Communications Letters, vol. 21, no. 8, pp. 1847-1850, 2017.

[6] P. Sui, Y. Guo, K.-F. Zhang, and H. Li, "Frequency-hopping transmitter fingerprint feature classification based on kernel collaborative representation classifier," Wireless Communications and Mobile Computing, vol. 2017, Article ID 9403590, 9 pages, 2017.

[7] Z. Tang and Y.-K. Lei, "Radio transmitter identification based on collaborative representation," Wireless Personal Communications, vol. 96, no. 1, pp. 1377-1391, 2017.

[8] Z. Tang and Y. K. Lei, "Method of individual communication transmitter identification based on maximum correntrogy," Journal of Communications, vol. 37, no. 12, pp. 171-175, 2016.

[9] D. F. Ren, T. Zhang, J. Han et al., "Specific emitter identification based on ITD and texture analysis," Journal of Communications, vol. 38, no. 12, pp. 160-168, 2017.
[10] J. Han, T. Zhang, H. H. Wang et al., "Communication emitter individual identification based on 3D-Hilbert energy spectrum and multi-scale fractal features," Journal of Communications, vol. 38, no. 4, pp. 99-109, 2017.

[11] D. M. Vo and S.-W. Lee, "Robust face recognition via hierarchical collaborative representation," Information Sciences, vol. 432, pp. 332-346, 2017.

[12] I. J. Goodfellow, D. Warde-Farley, M. Mirza et al., "Maxout networks," Computer Science, pp. 1319-1327, 2013.

[13] F. Schroff, D. Kalenichenko, and J. Philbin, "FaceNet: a unified embedding for face recognition and clustering," in Proceedings of the IEEE Conference on Computer Vision and Pattern Recognition (CVPR '15), pp. 815-823, IEEE, Boston, Mass, USA, June 2015.

[14] K. Nigam, A. K. Mccallum, S. Thrun, and T. Mitchell, “Text classification from labeled and unlabeled documents using EM," Machine Learning, vol. 39, no. 2, pp. 103-134, 2000.

[15] D. J. Miller and H. S. Uyar, "A mixture of experts classifier with learning based on both labelled and unlabelled data," in Proceedings of the advances in Neural Information Processing System 9 (NIPS), pp. 571-577, MIT Press, Cambridge, Mass, USA, 1997. 


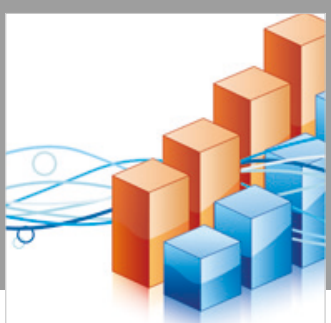

Advances in

Operations Research

\section{-n-m}
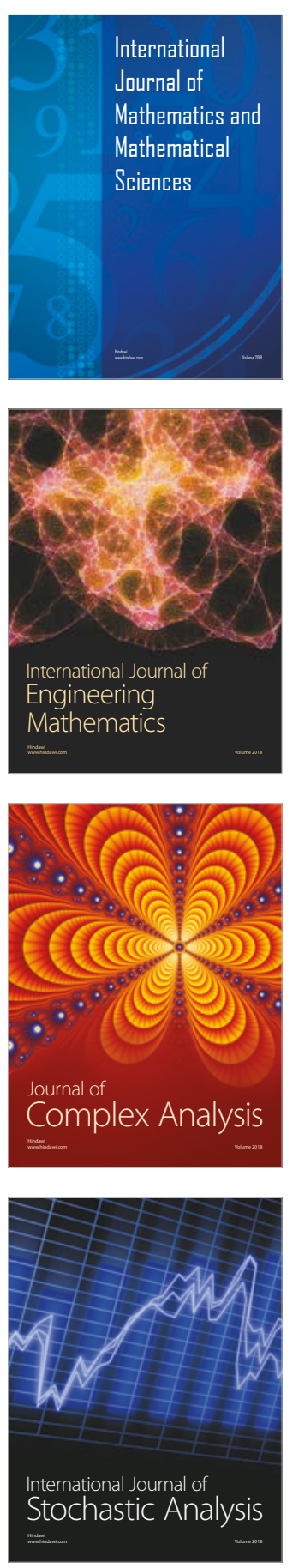
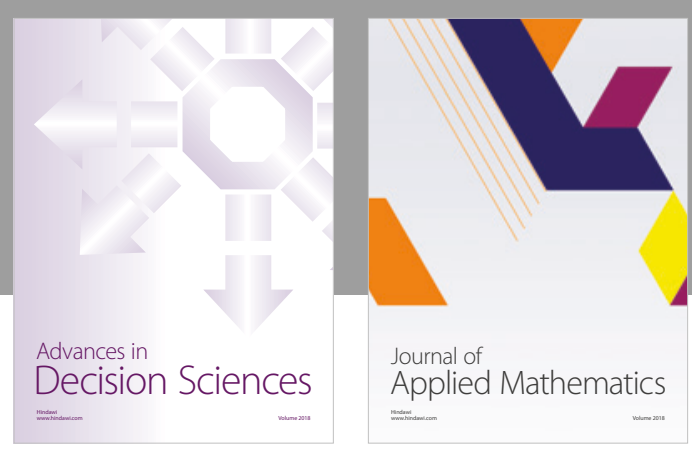

Journal of

Applied Mathematics
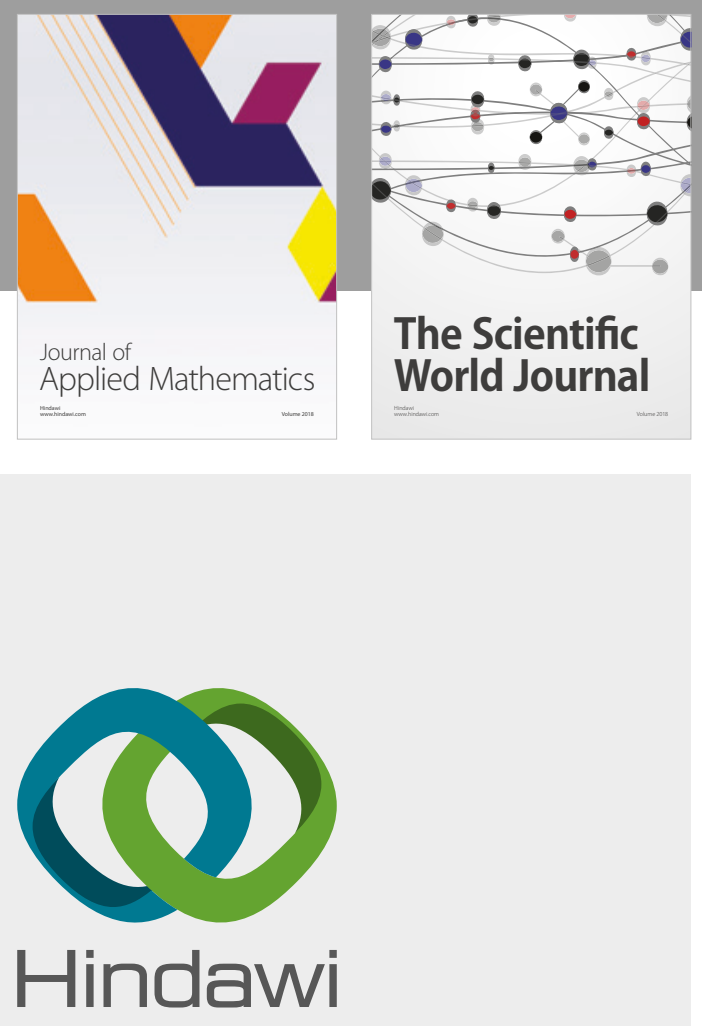

Submit your manuscripts at

www.hindawi.com

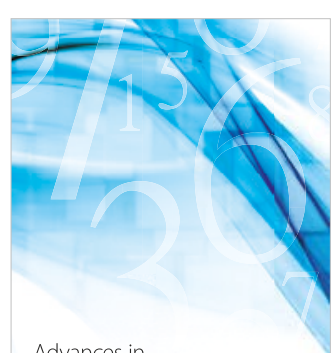

Advances in
Numerical Analysis
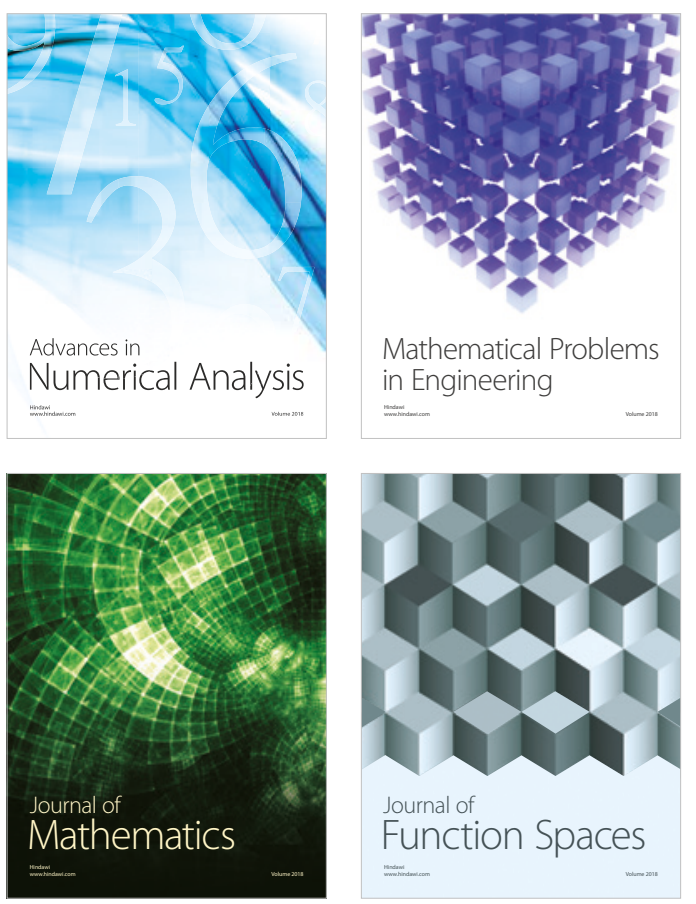

Mathematical Problems in Engineering

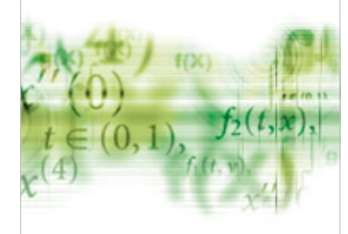

International Journal of

Differential Equations

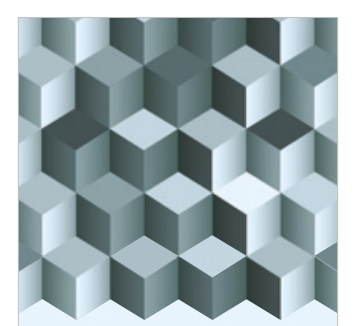

Journal of

Function Spaces

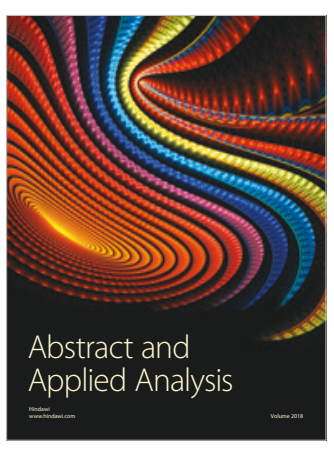

The Scientific

World Journal

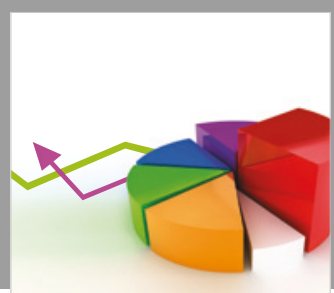

Journal of

Probability and Statistics
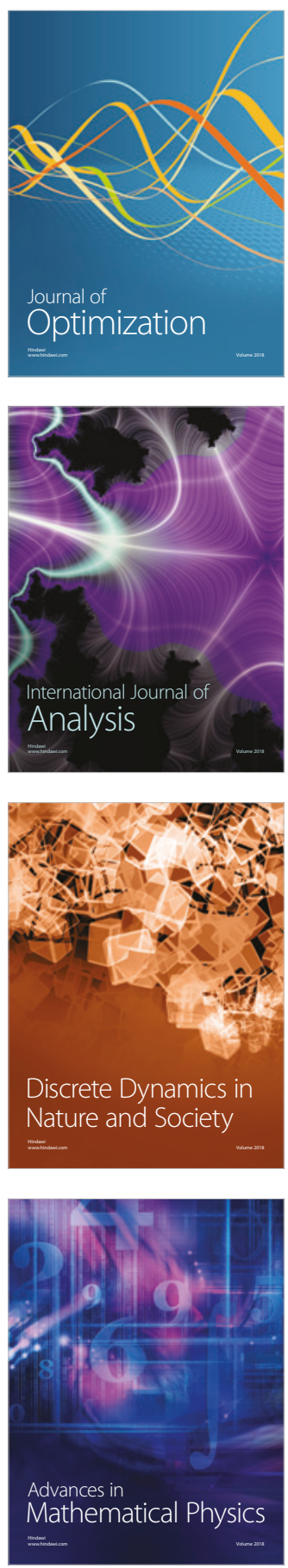